\title{
Least-squares Reverse Time Migration of Marine Data with Frequency-selection Encoding Wei Dai ${ }^{1}$, Yunsong Huang, ${ }^{2}$ and Gerard T. Schuster ${ }^{2}$ \\ ${ }^{1}$ Formerly at King Abdullah University of Science and Technology; Presently at WesternGeco. \\ ${ }^{2}$ King Abdullah University of Science and Technology.
}

\section{SUMMARY}

The phase-encoding technique can sometimes increase the efficiency of the least-squares reverse time migration (LSRTM) by more than one order of magnitude. However, traditional random encoding functions require all the encoded shots to share the same receiver locations, thus limiting the usage to seismic surveys with a fixed spread geometry. We implement a frequency-selection encoding strategy that accommodates data with a marine streamer geometry. The encoding functions are delta functions in the frequency domain, so that all the encoded shots have unique non-overlapping frequency content, and the receivers can distinguish the wavefield from each shot with a unique frequency band. Since the encoding functions are orthogonal to each other, there will be no crosstalk between different shots during modeling and migration. With the frequency-selection encoding method, the computational efficiency of LSRTM is increased so that its cost is comparable to conventional RTM for both the Marmousi2 model and a marine data set recorded in the Gulf of Mexico. With more iterations, the LSRTM image quality is further improved. We conclude that LSRTM with frequency-selection is an efficient migration method that can sometimes produce more focused images than conventional RTM.

\section{INTRODUCTION}

The phase-encoding technique (Romero et al., 2000; Krebs et al., 2009; Schuster et al., 2011) has been applied to increase the computational efficiency of seismic modeling and migration and it can reduce the cost of LSRTM to the level of conventional RTM. However, the random encoding functions used by Romero et al. (2000), Krebs et al. (2009), Schuster et al. (2011) and Dai et al. (2012), cannot be easily applied to a seismic survey with a marine streamer geometry (Routh et al., 2011; Huang and Schuster, 2012) because, although the calculated synthetic data are of fixed spread geometry, the observed data are recorded with a marine streamer geometry. Huang and Schuster (2012) proposed a frequency-selection encoding strategy for least-squares phase shift migration, which is applicable to marine data.

The frequency-selection encoding strategy can also be applied to least-squares reverse time migration with the time-domain single frequency simulation method proposed by Nihei and $\mathrm{Li}$ (2007), where the time-domain simulations are performed with a single-frequency source instead of the conventional broadband source, so that the single-frequency response of the medium to a point source can be extracted from the wavefield after it reaches steady state. Compared to the conventional frequency- domain director solver, their method has significantly lower arithmetic complexity and storage requirements in the 3D case (Nihei and Li, 2007; Sirgue et al., 2008). This method is also capable of modeling multiple sources at the same time without generating any crosstalk once each shot is assigned a different frequency.

With frequency-selection encoding, many shots (limited by the number of discrete frequency samples) can be modeled and migrated during one wavefield propagation with a finitedifference solution to the wave equation. The selected frequencies can be changed from one iteration to another such that the full frequency content of each shot gather has been migrated at the end of iterations.

\section{THEORY}

A time-domain seismic data set $d\left(t, \mathbf{x}_{g}, \mathbf{x}_{s}\right)$ can be digitized into a $3 \mathrm{D}$ array $\mathbf{d}_{i t, i g, i s}\left(i t=1,2, . ., n_{t} ; i g=1,2, . ., n_{g} ; i s=1,2, . ., n_{s}\right)$, assuming there are $n_{t}$ time samples, $n_{g}$ receivers for a shot, and $n_{s}$ shots in total. Defining the time sampling interval as $d t$, the data can be transformed into the frequency domain as $\tilde{\mathbf{d}}_{i \omega, i g, i s}\left(i \omega=1,2, . ., n_{\omega}\right)$ with the angular frequency sampling is $d \omega=\frac{2 \pi}{n_{t} * d t}$. In the frequency domain, only these samples that fall into the frequency band of the seismic data are kept, so for a data set with maximum frequency $f_{\max }, n_{\omega}$ can be calculated as $n_{\omega}=\frac{2 \pi * f_{\max }}{d \omega}$.

The encoding process is similar to conventional blended source technique. All the shots are encoded with the encoding functions first, with the frequency-selection encoding function defined as

$$
N_{s}\left(i \omega, i \omega_{s}\right)=\left\{\begin{array}{l}
1 \quad \text { when } i \omega=i \omega_{S} \\
0 \quad \text { otherwise }
\end{array}\right.
$$

where $i \omega_{s}$ is a function of shot index is, and it represents the selected frequency for the shot. Then, all the shots are blended together to form the supergather

$$
\tilde{\mathbf{d}}_{i \omega_{s}, i g}=\sum_{i s=1}^{n_{s}} N_{S}\left(i \omega, i \omega_{s}\right) \tilde{\mathbf{d}}_{i \omega, i g, i s} .
$$

Now the supergather $\tilde{\mathbf{d}}_{i \omega_{s}, i g}$ becomes a $2 \mathrm{D}$ array, and each frequency component corresponds to a different shot. It is obvious that the frequency-selection encoding method is applicable to seismic data with a marine streamer acquisition geometry, because at each receiver position, the data components from different shots can be distinguished from one another according to their frequency content. With frequency-selection encoding, a supergather can only accommodate $n_{\omega}$ shots.

In LSRTM, the Born modeling operator is used to fit the observed reflection data with a reflectivity model $m(\mathbf{x})$. Following Symes and Carazzone (1991); Dai et al. (2012), the Born 


\section{Frequency-selection LSRTM}

modeling of a supergather can be computed in the time domain as

$$
\begin{gathered}
\left(\nabla^{2}-\frac{1}{c_{o}^{2}} \frac{\partial^{2}}{\partial t^{2}}\right) p_{o}(t, \mathbf{x})=-\sum_{s} \operatorname{Re}\left[W\left(\omega_{s}\right) e^{i \omega_{s} t}\right] \delta\left(\mathbf{x}-\mathbf{x}_{S}\right) . \\
\left(\nabla^{2}-\frac{1}{c_{o}^{2}} \frac{\partial^{2}}{\partial t^{2}}\right) \delta p(t, \mathbf{x})=m(\mathbf{x}) \frac{1}{c_{o}^{2}} \frac{\partial^{2} p_{o}(t, \mathbf{x})}{\partial t^{2}}
\end{gathered}
$$

The frequency-domain data $\tilde{d}\left(\omega_{s}, \mathbf{x}_{g}\right)$ can be extracted from $\delta p\left(t, \mathbf{x}_{g}\right)$ according to Nihei and Li (2007)

$$
\tilde{d}\left(\omega_{s}, \mathbf{x}_{g}\right)=\frac{1}{T} \int_{T}^{2 T} \delta p\left(t, \mathbf{x}_{g}\right) e^{-i \omega_{s} t} d t .
$$

The migration operator can be formulated as the adjoint of the Born modeling operator. Corresponding to equations 4 and 5, the migration formula is

$$
\begin{gathered}
\left(\nabla^{2}-\frac{1}{c_{o}^{2}} \frac{\partial^{2}}{\partial t^{2}}\right) q(t, \mathbf{x})=-\sum_{\omega_{s}} \sum_{g} \operatorname{Re}\left[\tilde{d}\left(\omega_{s}, \mathbf{x}_{g}\right) e^{i \omega_{s} t}\right] \delta\left(\mathbf{x}-\mathbf{x}_{g}\right), \\
m(\mathbf{x})=\int_{T}^{2 T} \frac{1}{c_{o}^{2}} \frac{\partial^{2} p_{o}(t, \mathbf{x})}{\partial t^{2}} \times q(t, \mathbf{x}) d t
\end{gathered}
$$

where $p_{o}(t, \mathbf{x})$ is the source wavefield obtained with equation $3, q(t, \mathbf{x})$ is the receiver wavefield, and equation 7 indicates a zero lag imaging condition after both the source and receiver wavefields reach steady state.

With the above definitions of the Born modeling and migration operators, the misfit functional

$$
f(\mathbf{m})=\frac{1}{2}\|\mathbf{L} \mathbf{m}-\mathbf{d}\|^{2}
$$

can be minimized to find a reflectivity model $\mathbf{m}$ that best fits the observed data $\mathbf{d}$ with the Born modeling operator $\mathbf{L}$. At each iteration, a new supergather with new encoding functions should be used to sample a different frequency for each shot.Therefore, if $I$ frequencies are needed to avoid wrap-around effects, the LSM procedure should be iterated at least $I$ iterations to ensure that all the frequencies are visited by a shot.

\section{NUMERICAL RESULTS}

The proposed method is tested on data associated with the Marmousi2 model, where the original model is modified to be the dimensions of $8 \mathrm{~km} \times 3.5 \mathrm{~km}$ with a $10 \mathrm{~m}$ grid interval (Figure 1a). The synthetic data are generated with a marinestreamer geometry, where 400 shots are excited with a $20 \mathrm{~m}$ offset interval at the depth of $10 \mathrm{~m}$. Each shot is recorded with a $2 \mathrm{~km}$ long cable with 201 receivers and $10 \mathrm{~m}$ interval. The minimum source-receiver offset is zero meters and the maximum offset is $2 \mathrm{~km}$. A Ricker wavelet with a $20-\mathrm{Hz}$ peak frequency is used as the source wavelet. The record length is $8 \mathrm{sec}$ in time and a 2-8 finite-difference code is used for the implementation of RTM.

Due to data redundancy, the frequency-domain data can be represented by a coarser sampling than the Nyquist rate. According to Mulder and Plessix (2004b), the maximum necessary frequency sampling is estimated to be $0.70 \mathrm{~Hz}$ in this example. We choose $0.625 \mathrm{~Hz}$ as the frequency sampling for convenience, and thus the range of $0-50 \mathrm{~Hz}$ can be represented by 80 frequency samples. The frequency-selection encoding scheme is as follows. Each shot is assigned one out of 400 frequencies, and then blended to form a supergather. For the next iteration, the frequency assigned to each shot is increased by $0.625 \mathrm{~Hz}$. When the frequency of a shot exceeds $50 \mathrm{~Hz}$, it will be wrapped around to the low frequencies. Therefore, 80 such LSRTM iterations can sample the frequency domain of interest. Please note that in the end of 80 iterations, 80 frequency data samples are migrated for each shot.

A pseudo-spectral method is used to compute the true data of 400 CSGs.The LSRTM method is first applied to those 400 shot gathers without encoding based on the migration velocity in Figure 1b. Figure 2a shows the conventional RTM image after illumination compensation (Mulder and Plessix, 2004a) and Figure $2 \mathrm{~b}$ shows the LSRTM image after 15 iterations. Compared to the conventional RTM image, the LSRTM image contains much fewer artifacts (e.g., the back-scattering artifacts in the shallow zone), shows better continuity for reflector amplitudes, and is of higher spatial resolution.

Those 400 shot gathers are then transformed into the frequency domain and 80 supergathers are formed with the above frequencyselection encoding strategy. Those 80 supergathers are migrated with the same velocity in Figure $1 \mathrm{~b}$ and stacked together. This method was referred to as iterative stacking method, which sometimes can be cheaper than conventional RTM (Schuster et al., 2011). The image is shown in Figure 2c and it is almost identical to the conventional shot domain RTM image in Figure 2a, except that some high-frequency noise are present.

To reduce the migration artifacts and improve the image resolution, the same 80 supergathers are migrated with the LSRTM algorithm with one supergather for each iteration. Figure $3 \mathrm{a}$ plots the image for the first iteration, which contains strong ringing artifacts. As iterations proceed, the LSRTM image quality gradually improves (see Figure $3 \mathrm{~b}$ for the 20-iteration result). Figure $3 \mathrm{c}$ shows the image after 80 iterations which is of higher resolution than the conventional RTM image in Figure 2a. The drawback is that there is high-frequency noise in the frequency-selection LSRTM image compared to the one without encoding. In this example, the LSRTM image without encoding is of 30 times the cost of conventional RTM, and the frequency-selection LSRTM image is of 3.2 times the cost of conventional RTM. Therefore, the frequency-selection encoding increases the computational efficiency by 9.4 times.

The frequency-selection LSRTM method is also tested on a 2D marine data set. There are 496 shots with a shot interval of $37.5 \mathrm{~m}$. Each shot is recorded by a $6 \mathrm{~km}$ long cable with 480 receivers at a receiver interval of $12.5 \mathrm{~m}$. The nearest offset is $198 \mathrm{~m}$. Each trace is multiplied by $\sqrt{i / \omega}$ in the frequency domain and then scaled by $\sqrt{t}$ in the time domain to correct for 3D geometrical spreading (Zhou et al., 1997). Then, the CSGs are Wiener filtered to transform the original wavelet to a Ricker wavelet with a $25-\mathrm{Hz}$ peak frequency. The original wavelet is estimated by stacking traces with a strong waterbottom reflection, and windowing the water-bottom reflection 


\section{Frequency-selection LSRTM}

event.

The data set is first migrated with the conventional shot-domain RTM method with a migration velocity that is obtained by waveform inversion (Boonyasiriwat et al., 2010), and the image is shown in Figure 4a. It is seen that there are strong artifacts near the shallow reflectors, which are caused by head waves and diving waves (Liu et al., 2011). In the bottom right corner of the image, there are low-frequency horizontal stripes.

According to Mulder and Plessix (2004b), the maximum frequency sampling for this field data set is larger than $1 \mathrm{~Hz}$, assuming an average water depth of $0.5 \mathrm{~km}$ and a maximum depth of $2.5 \mathrm{~km}$. However, due to the fact that the shot spacing $37.5 \mathrm{~m}$ is larger than half of the dominant wavelength (30 $\mathrm{m}$ ) in the water, a denser sampling is needed. Empirical tests suggest a frequency sampling of $0.3 \mathrm{~Hz}$. Therefore, with 208 iterations, 208 unique frequencies are used by any one shot. To form a supergather, all the shots are encoded with unique frequency-selection functions and blended together. For the next iteration, the frequency of each shot is shifted by $0.3 \mathrm{~Hz}$ to form a new supergather. The LSRTM algorithm iterates for 208 iterations to cover all the source frequencies and the image is shown in Figure $4 \mathrm{~b}$ (The white and black boxes show the areas for zoom views). The low-frequency artifacts in the shallow part and in the right bottom corner are removed. Furthermore, the resolution of the LSRTM image is enhanced compared to the shot-domain RTM image, which leads to better delineation of the faults as shown in the zoom views in Figures 5 and 6 . The drawback is that there is still some highfrequency noise present in the LSRTM image. In this example, the computational cost of the frequency-selection LSRTM image is about 5 times of that of the conventional RTM.

\section{CONCLUSION}

In this paper, we implemented a frequency-selection encoding strategy to speed up the least-squares reverse time migration of marine data. The traditional random phase encoding method is not applicable to marine data due to the mismatch in acquisition geometry between the observed data and the calculated synthetic data. With frequency-selection encoding, all the shots are encoded with encoding functions that are orthogonal to each other in the frequency domain, so the calculated synthetic data can be effectively decoded at the receiver locations for comparison with the observed data. Because of the data redundancy in the frequency domain, the frequency sampling rate can be large, which leads to significant computational savings. Numerical tests on the Marmousi 2 model and a field data set from the Gulf of Mexico show that frequencyselection encoding can significantly improve the efficiency of the LSRTM and reduce its cost to the level of conventional shot domain RTM. Empirical results suggest that the LSRTM with frequency-selection encoding is an efficient method to produce better images than conventional RTM.

The drawback of the frequency-selection LSRTM method is that it is sensitive to errors in the velocity model. With frequencyselection encoding, LSRTM inverts a small part of the data at every iteration. When the migration velocity is not accurate, the reflectivity model that explains each small part of the data can be different from one another. Therefore, the final stack can become blurred and defocused.

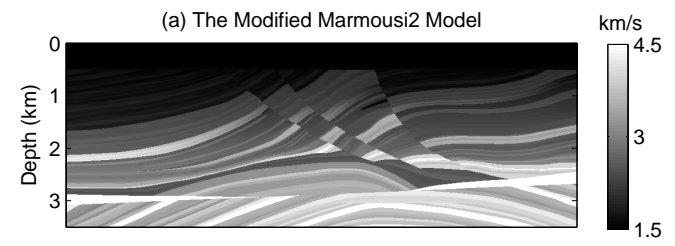

(b) The Smooth Migration Velocity

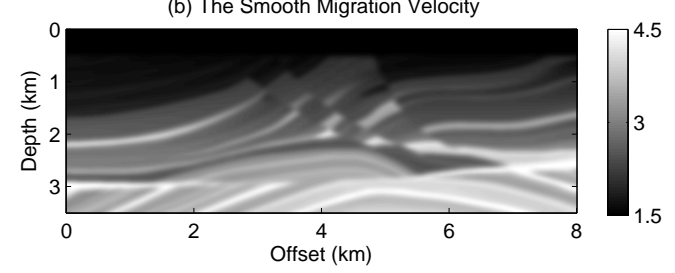

Figure 1: The Marmousi2 model: (a) modified Marmousi2 velocity model and (b) smooth migration velocity model.

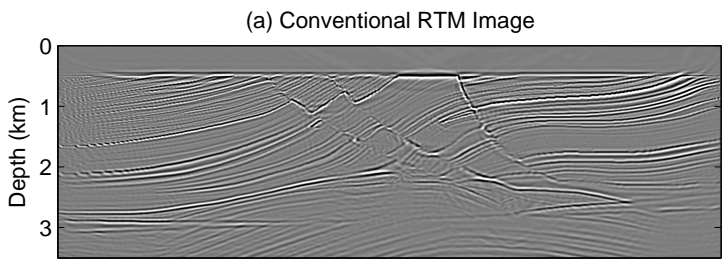

(b) LSRTM Image without Encoding

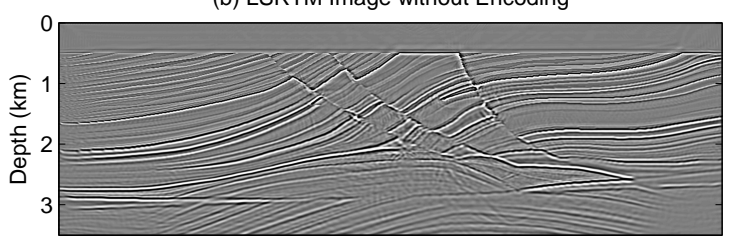

(C) Iterative Stacking Image

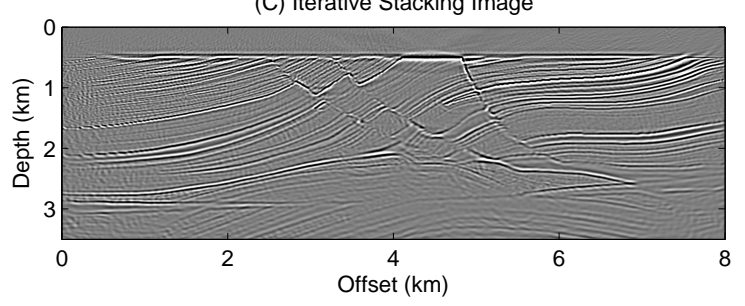

Figure 2: Migration images obtained by (a) conventional shotdomain RTM, (b) LSRTM without encoding, and (c) the iterative stacking methods. 
(a) LSRTM Image after the 1st Iteration

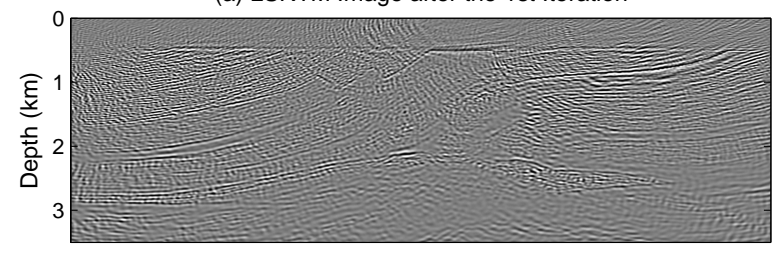

(b) LSRTM Image after 20 Iterations

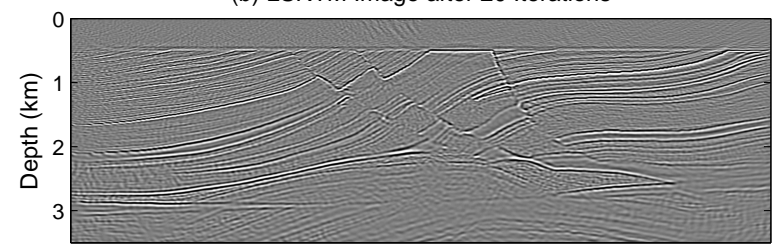

(c) LSRTM Image after 80 Iterations

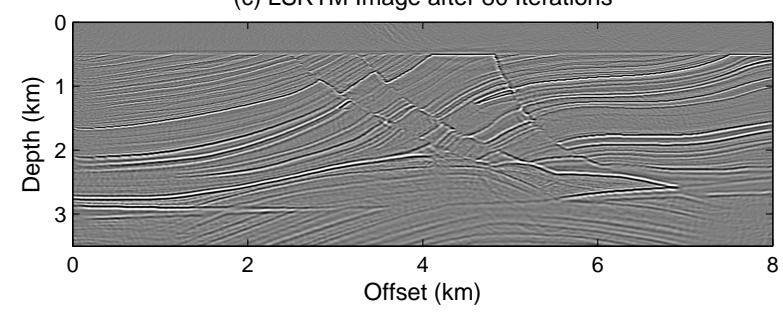

Figure 3: The frequency-selection LSRTM images after (a) 1 iteration, (b) 20 iterations, and (c) 80 iterations.

(a) Shot-domain RTM Image

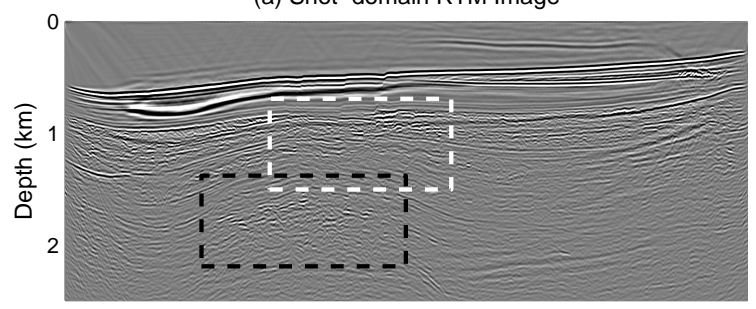

(b) Frequency-selection LSRTM Image

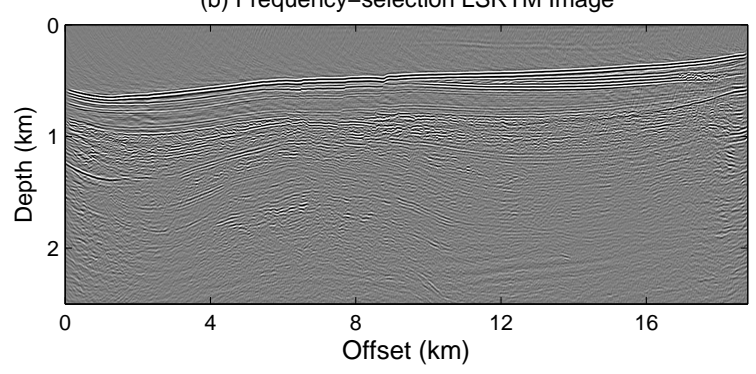

Figure 4: The migration images obtained with the (a) conventional RTM and (b) frequency-selection LSRTM methods. White and black boxes indicate the areas for zoom views. (a) Shot-domain RTM Image

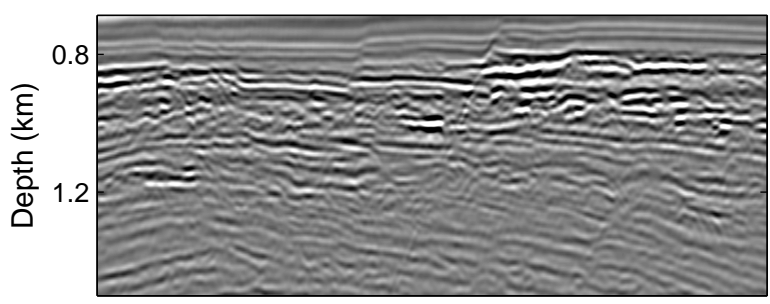

(b) Frequency-selection LSRTM Image

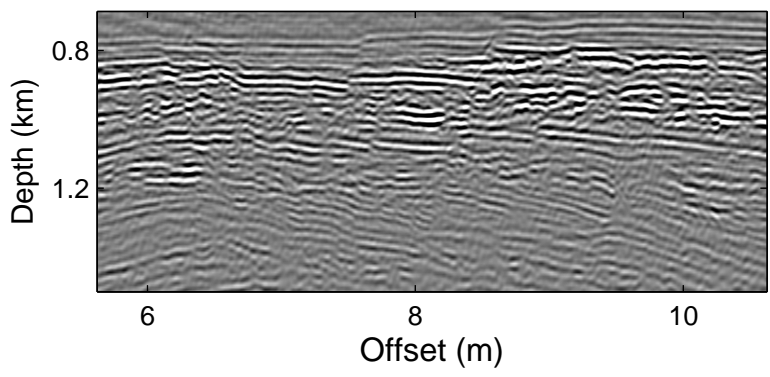

Figure 5: The zoom view of the white box for the (a) conventional RTM and (b) frequency-selection LSRTM images.

(a) Shot-domain RTM Image

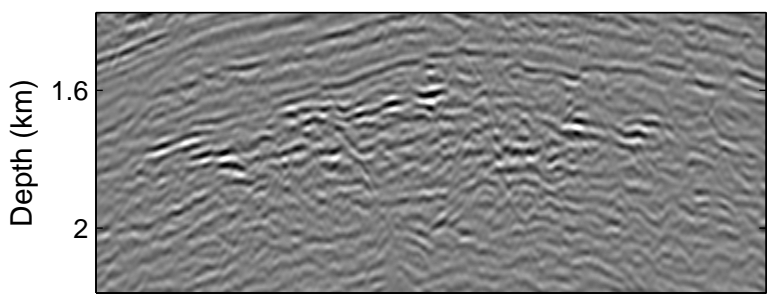

(b) Frequency-selection LSRTM Image

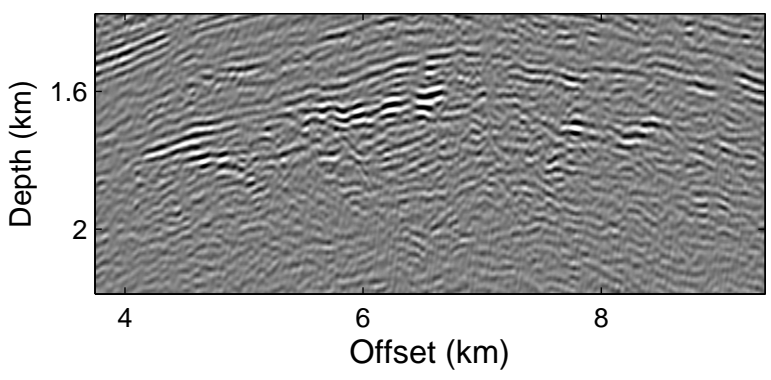

Figure 6: The zoom view of the black box for the (a) conventional RTM and (b) frequency-selection LSRTM images. 
http://dx.doi.org/10.1190/segam2013-0412.1

\section{EDITED REFERENCES}

Note: This reference list is a copy-edited version of the reference list submitted by the author. Reference lists for the 2013 SEG Technical Program Expanded Abstracts have been copy edited so that references provided with the online metadata for each paper will achieve a high degree of linking to cited sources that appear on the Web.

\section{REFERENCES}

Boonyasiriwat, C., G. T. Schuster, P. Valasek, and W. Cao, 2010, Applications of multiscale waveform inversion to marine data using a flooding technique and dynamic early-arrival windows: Geophysics, 75, no. 6, R129-R136, http://dx.doi.org/10.1190/1.3507237.

Dai, W., P. Fowler, and G. T. Schuster, 2012, Multisource least-squares reverse time migration: Geophysical Prospecting, 60, 681-695, http://dx.doi.org/10.1111/i.1365-2478.2012.01092.x.

Huang, Y., and G. T. Schuster, 2012, Multisource least-squares migration of marine streamer and land data with frequency-division encoding: Geophysical Prospecting, 60, 663-680, http://dx.doi.org/10.1111/i.1365-2478.2012.01086.x.

Krebs, J. R., J. E. Anderson, D. Hinkley, R. Neelamani, S. Lee, A. Baumstein, and M.-D. Lacasse, 2009, Fast full-wavefield seismic inversion using encoded sources: Geophysics, 74, no. 6, WCC177WCC188, http://dx.doi.org/10.1190/1.3230502.

Liu, F., G. Zhang, S. A. Morton, and J. P. Leveille, 2011, An effective imaging condition for reverse-time migration using wavefield decomposition: Geophysics, 76, no. 1, S29-S39, http://dx.doi.org/10.1190/1.3533914.

Mulder, W. A., and R.-E. Plessix, 2004a, A comparison between one-way and two-way wave-equation migration: Geophysics, 69, 1491-1504, http://dx.doi.org/10.1190/1.1836822.

Mulder, W. A., and R.-E. Plessix, 2004b, How to choose a subset of frequencies in frequency-domain finite-difference migration: Geophysical Journal International 158, 801-812, http://dx.doi.org/10.1111/j.1365-246X.2004.02336.x.

Nihei K. T., and X. Li, 2007, Frequency response modelling of seismic waves using finite difference time domain with phase sensitive detection (TD-PSD): Geophysical Journal International, 169, 1069-1078, http://dx.doi.org/10.1111/j.1365-246X.2006.03262.x.

Romero, L. A., D. C. Ghiglia, C. C. Ober, and S. A. Morton, 2000, Phase encoding of shot records in prestack migration: Geophysics, 65, 426-436, http://dx.doi.org/10.1190/1.1444737.

Routh, P., J. Krebs, S. Lazaratos, A. Baumstein, I. Chikichev, S. Lee, N. Downey, D. Hinkley, and J. Anderson, 2011, Full-wavefield inversion of marine streamer data with the encoded simultaneous source method: 73rd Conference \& Exhibition, EAGE, Extended Abstracts, F032.

Schuster, G. T., X. Wang, Y. Huang, W. Dai, and C. Boonyasiriwat, 2011, Theory of multisource crosstalk reduction by phase-encoded statics: Geophysical Journal International, 184, 1289-1303, http://dx.doi.org/10.1111/j.1365-246X.2010.04906.x.

Sirgue, L., J. T. Etgen, and U. Albertin, 2008, 3D frequency domain waveform inversion using time domain finite difference methods: 70th Conference \& Exhibition, EAGE, Extended Abstracts, F022.

Symes, W. W., and J. J. Carazzone, 1991, Velocity inversion by differential sembla nce optimization: Geophysics, 56, 654-663, http://dx.doi.org/10.1190/1.1443082. 
Zhou, C., G. T. Schuster, S. Hassanzadeh, and J. M. Harris, 1997, Elastic wave equation traveltime and waveform inversion of crosswell data: Geophysics, 62, 853-868, http://dx.doi.org/10.1190/1.1444194. 